\title{
¿Resistirá la ética de la abogacía la presión de los cambios de la profesión?: Abogacía y ética en el siglo XXI
}

\section{Horacio M. LYNCH²}

El forzoso encierro por la pandemia fue una oportunidad para escribir una obra colectiva intentando responder preguntas claves respecto de la ética de la abogacía: su efectivo cumplimiento por los profesionales, los grandes cambios que se están produciendo en la profesión en todo el mundo y cómo pueden impactar en las reglas que rigen esta delicada actividad.

Es que la segunda década del siglo XXI encuentra a la profesión en pleno cambio por la tecnología y por la forma como se prestan de los servicios legales, $y$, también, respecto de la educación legal y la formación de abogados.

Los cambios que la tecnología ha provocado en la profesión son espectaculares en todo sentido, positivos y negativos, y afectan la forma como se ejerce y también como se prestan los servicios legales. La inteligencia artificial ha impactado en el corazón del trabajo del abogado que antes destinaba buena parte de su tiempo a la investigación y a la revisión ABOGACIA E T I C A SIGLO XXI de instrumentos legales (IA), a las reuniones y audiencias (teleconferencias). Otro gran cambio es que se ha diluido la importancia de la ubicación del abogado, del país en el que se encuentra físicamente. Por otro lado, la prestación de los servicios ha impactado en las organizaciones: pensar que no hace mucho se comenzó a hablar del legal bussines, en tanto hoy se habla de la legal industry. Oficinas jurídicas grandes en los '80 eran a las de más de 100 abogados: ¡hoy estamos hablando de 10 veces más!

En lo relativo a la formación, hace tiempo en la Comunidad Europea se determinó que nadie puede ser abogado sin dar un examen de habilitación ante los colegios profesionales (antigua práctica en los países anglosajones que, desde siempre, separaron los estudios de Derecho de la formación de abogados; el gran presidente y gran abogado Abraham Lincoln nunca fue a una Facultad de Derecho). Una vez más, en estas latitudes, parecemos congelados en el tiempo.

Esta obra en colaboración reúne miradas y reflexiones de experimentados abogados sobre la abogacía y la ética ante los nuevos retos de la profesión con énfasis en la realidad del ejercicio de la abogacía en la Argentina y el relativo cumplimiento de las normas éticas. Pero la mayoría de quienes escriben tienen, además, una marcada inclinación

1 Nota del libro ABOGACÍA Y ÉTICA EN EL SIGLO XXI -EBook - 1a. Edición digital, Mayo 2021. Horacio M. LYNCH et al. DESCARGA GRATUITA en Varios formatos (PDF, eBook, Kindle) https:/ / foresjusticia.org/2021/05/13/abogacia-y-etica-en-elsiglo-xxi/

2 El autor que ha dirigido la obra junto con Marcelo Gobbi, tiene cincuenta años de ejercicio profesional y casi tantos de actuación en instituciones nacionales e internacionales de la abogacía y de la Justicia. En 1976 fundó y presidió la institución convocante - FORES (Foro de Estudios sobre la Administración de Justicia)durante veinte años - la primera ONG de América Latina sobre la Justicia. 
por la docencia y por la investigación. A sus reflexiones se suman los resultados de una encuesta realizada entre abogados jóvenes.

\section{Destinatarios del libro}

Son destinatarios de esta obra aquellos que se interesan por la abogacía, que la ejercen y la consideran una profesión apasionante, que ven claramente a los abogados trabajando para la Justicia de uno u otro lado del mostrador. También, es para quienes proclaman la importancia de la profesión para traer paz al mundo, para facilitar el desarrollo de los países, para afirmar una convivencia republicana y democrática; aquellos, en fin, que comparten la visión de Lincoln de que el abogado, como pacificador, componedor, tiene una sublime oportunidad de ser un buen hombre. Está escrito también para los que, aun dudando que la profesión deba ser ejercida de una determinada forma y bajo ciertas reglas, aspiran siempre a lo mejor. Es para los que se preocupan por los avances tecnológicos, por los cambios en la forma cómo se ejerce la abogacía o cómo se proveen los servicios legales y que presienten que hay nuevos riesgos que involucran los principios más elementales de la profesión. La variedad de enfoques y de temas debería ayudar a que el lector encontrara por sí mismo respuestas a sus inquietudes. Sirve, en fin, para la enseñanza, para mostrar la ética profesional desde distintos aspectos, para cuestionar, debatir, para leerlo escuchando los sabios consejos de experimentados abogados que aman la profesión y que entienden que es posible - y conveniente - ejercerla según 'las reglas del arte'.

\section{Objetivos}

La obra se emprendió con múltiples objetivos en derredor de aquellas preguntas iniciales: cómo es el cumplimiento efectivo de las normas de ética en el mundo y en la Argentina, profundizando las causas de esta situación y la enseñanza de la ética en dicho país. Busca analizar los cambios se están ocurriendo en la profesión y su impacto en las reglas deontológicas. Pretende también revisar la vigencia de los principios generales, ahondar algunos temas éticos muy delicados y relacionar todo con la ética judicial. Mi comentario seguirá este esquema, aunque la estructura del libro, por razones metodológicas, sea diferente.

\section{La realidad de la ética profesional}

Inspira el libro la convicción de que en el mundo hay una gran distancia entre los principios que rigen la profesión y la realidad, distancia que es muy grave en Argentina, en buena parte porque las autoridades responsables - Colegios de Abogados, las Facultades de Derecho y la Justicia - han descuidado la cuestión. Para corroborar la situación en Argentina se utilizan los resultados de una encuesta entre abogados 
jóvenes y se confrontan esta visión con la situación de otros países para tener puntos de comparación.

Para intentar bucear más profundamente en la realidad de la Argentina el suscripto realizó una encuesta entre abogados jóvenes, cuyos resultados abonan su capítulo La ética de la abogacía en la Argentina 2020 (y los resultados de una encuesta), en el que se analizar los resultados. Además repasa la crisis de la abogacía instalada desde hace varias décadas en la Argentina sin síntomas de mejoría, estimando que la situación crítica es consecuencia directa de la ausencia de enseñanza de la ética de la abogacía y la poca importancia asignada el tema en los organismos responsables. Como los estudios comparativos ayudan a dimensionar la situación, el libro incluye dos estudios sobre la realidad en otros países. En el primero, tres destacados profesionales que ejercen en Francia - Beatriz Burghetto, Christophe Dubois y Santiago Muzio de Place - abordan La ética profesional del abogado en Francia, describiendo los requisitos para convertirse en abogado en ese país, en particular, la preparación de candidatos a abogado en las escuelas de abogados francesas con énfasis en la enseñanza de la ética profesional. Pero también incluyen referencias a la aplicación en la práctica de las reglas de ética con citas a un interesante estudio empírico y con las opiniones y experiencias personales de los autores. Y otro capítulo de Melisa Ortes González, abogada argentina que ejerce en una importante firma de Nueva York, La Ética Profesional en los Estados Unidos de América, en el que afirma que para comprender el rol que tienen estas normas en el foro local americano hay que considerar el contexto histórico de su desarrollo desde la sanción de la Constitución de los Estados Unidos y que se propagó y reafirmó con el advenimiento de las asociaciones profesionales en cada uno de los estados. Los resultados del estudio preliminar y de la encuesta realizada entre jóvenes profesionales sobre la realidad del cumplimiento de las normas éticas en la Argentina, no sólo es preocupante, sino que, confrontada con la de los EE.UU. y Francia, lo es aún más.

\section{Nueva abogacía, ¿nuevos dilemas éticos?}

La profesión se está transformando radicalmente en el mundo, con cambios que tarde o temprano llegarán a estas orillas y en el libro se analiza su impacto en tanto pueden implicar nuevos dilemas éticos. También se lo vincula con las nuevas visiones que habrá que tener respecto de la preparación de los abogados, y la forma como se enseña en la Argentina.

En su ensayo Compromisos relevantes para la práctica de la abogacía actual el Dr. ArmandoS. Andruet [h] (ex magistrado y doble Académico de Derecho y de Medicina) propone cinco criterios a tener en cuenta sugiriendo la manera en que las Universidades, los colegios de abogados y tribunales de disciplina pueden enfrentarlos. Señala que las Universidades no se hacen cargo de la explicación de la ética profesional en concreto pues sólo presentan una teorización por lo que también deben atender a este cuestionamiento. Los colegios de abogados deberían, por su parte, construir una conciencia profesional. Y corresponde que los tribunales de disciplina fortalezcan la difusión de sus resoluciones para, con ello, profundizar la ejemplaridad, insistiendo en la matriz ética de la profesión. Por su parte, Ricardo Rojas, ex magistrado, investigador y catedrático, propone reflexionar sobre un 
distinto enfoque de la formación, Del abogado litigante al abogado negociador, un necesario cambio de paradigma, donde, con ayuda del análisis económico del Derecho pondera en especial, en la labor del letrado en su función de promotor de soluciones eficientes a los conflictos, más que como litigante sobre cuestiones legales, proponiendo sumar a su formación las habilidades como negociador y empresario para ofrecer un mejor asesoramiento a su cliente. María Julia Fornari, docente y abogada con sólida experiencia en mediación nos brinda sus Reflexiones el ejercicio profesional y la tecnología, en el que muestra el contraste entre los vertiginosos adelantos de la tecnología que impactan en todas las áreas de nuestra vida cotidiana con el ejercicio de la profesión de abogado en un país anclado en el pasado. Siguiendo esta línea, en Nueva abogacía ¿nuevos dilemas éticos?, el suscripto intenta dar respuesta a la cuestión de si los cambios en la profesión han determinados nuevos problemas éticos para los abogados, o son los de siempre, aunque presentados en odres nuevos. Tres ensayos muy desafiantes completan este panorama: dos de Marcelo Gobbi, destacado abogado corporativo - Nuevos [ya no tanto) paradigmas en la abogacía y Dónde diablos se ejerce la abogacía - que renuevan los planteos anteriores y analiza, en el primero, la necesidad de los abogados y sus organizaciones de responder al impacto de la automatización; en tanto en el segundo cuestiona la relativa utilidad de los criterios puramente territoriales, aplicados por las regulaciones sobre la abogacía como consecuencia de la deslocalización; y del investigador Demetrio Alejandro Chamatropulos, La innovación y el derecho en tiempos de pandemia, en el que llama la atención sobre los nuevos fenómenos que provoca la pandemia del COVID-19).

\section{La enseñanza de la ética de la abogacía}

Varios ensayos reflejan la preocupación de los autores por la mala o nula enseñanza de la ética profesional en las Facultades de Derecho y en los colegios profesionales de Argentina: si efectivamente se brinda, y, cuando se enseña, cómo se lo hace, cómo son sus planes y métodos, su orientación, su vinculación con la práctica diaria de la abogacía incluyendo nuevos enfoques, como el de comparar la enseñanza de la ética en las Facultades de Derecho con las de Medicina.

En la colaboración ¿No INTERESA LA ÉTICA?, Marina Iturbide destacada comercialista, reflexiona sobre las razones por las que no se enseña la ética profesional a todos los abogados egresados del país. Por su parte, en La enseñanza universitaria de la ética profesional en la argentina, Juan Marcos Pueyrredón titular de una cátedra de Ética de la Universidad Católica Argentina Santa María de Buenos Aires, junto con sus ayudantes Guillermina Federik y Lucía María Traverso, analizan cómo se enseña en las Facultades de Derecho en la Argentina (en las que la brindan) y señalan cómo se podría mejorar la situación. Y, como cierre, resalto el original estudio conjunto de Raúl Farías, director de los programas de formación de abogados de FORES, especializado en nuevas tecnologías, y de Lucía Beccar Varela, destacada médica, titulado Semejanzas y diferencias entre la ética de la abogacía y de la medicina. Nos dicen que "Todas las profesiones son importantes, pero sólo dos tienen en sus manos los bienes más preciados de las personas: la Medicina y el Derecho ... en el primer caso, los médicos cargan con la responsabilidad de la salud y de la vida de sus pacientes; en el segundo, los abogados con la libertad y el patrimonio de sus clientes". 


\section{Los principios y valores permanentes de la abogacía}

También se incluyen visiones que, apelando a los principios vigentes desde el origen de la profesión, analizan su vigencia actual, concluyendo que son más válidos que nunca, frescos y modernos, y con aptitud para afrontar los nuevos retos profesionales.

Dos profundos trabajos de Enrique V. del Carril, experto abogado, ex Presidente de FORES y del Colegio de Abogados de la Ciudad de Buenos Aires - Derecho, ética profesional y moral del abogado (importancia de la ética y moral profesional en la aplicación del derecho) - y otro Dos tradiciones jurídicas, una misma profesión nos muestran cómo la abogacía es la misma sin perjuicio de la tradición jurídica donde se ejerza y del Derecho de fondo que se aplique. Continúa un ensayo de Felipe Ordóñez, abogado y ex docente, sobre Perspectiva diacrónica del deber de verdad, en el que nos acerca a la idea del deber de verdad en el proceso judicial, recorriendo la interpretación que ha tenido el principio en algunos pueblos de la antigüedad hasta llegar a nuestros días. Muy interesante es el aporte de Damián F. Beccar Varela, fallecido ex titular del prestigioso estudio de Buenos Aires que lleva su apellido - Las nuevas reglas de ética profesional - con reflexiones sobre su trabajo de actualización de las reglas del Colegio de Abogados de la Ciudad de Buenos Aires.

\section{Abogacía penal y de familia, secreto profesional, honorarios y otros temas puntuales}

Para completar el panorama el libro incluye varios estudios sobre temas puntuales y estratégicos del ejercicio profesional, como la práctica en ciertas áreas complejas como familia y penal y el delicado tema del secreto profesional. Este tema también es abordado desde dos perspectivas, la inmutabilidad del secreto, y la colisión entre este principio y el de las normas sobre el lavado de dinero y el terrorismo. Revisa también el de los honorarios y el arancel obligatorio, terminando con dos trabajos sobre las realidades de los abogados externos e internos de las empresas.

En el primero, Juan María Rodríguez Estévez experimentado penalista, doctor en derecho y docente, aborda el tema de la Ética profesional y defensa penal eficaz, en el que plantea como una cuestión éticamente exigible para el abogado penalista el redoblar los esfuerzos por parte de la defensa para nivelar un escenario procesal de una desventaja inicial de fuerzas. Jorge A. Mazzinghi, ex Presidente de FORES y también profesor universitario, con destacada carrera profesional en el área del Derecho de familia nos brinda unas ricas páginas con sus Dilemas éticos habituales de los abogados que se dedican al derecho de familia, en las que explica el significado primordial de la Ética en el campo del Derecho de Familia basado en el carácter complejo o compuesto del interés del abogado que se dedica a atender esos asuntos que debe defender o resguardar. Pablo A. Pirovano prestigioso abogado con actuación en FORES y en el Colegio de Abogados de la Ciudad de Buenos Aires aborda la especificidad de los Principios éticos para los abogados árbitros que deben primar antes, durante y después de su actuación como árbitros jurisdiccionales, y las obligaciones de ser y mantener independencia e imparcialidad como contenedoras de los deberes éticos. El también reconocido penalista Hernán Munilla Lacasa en El abogado no puede ser obligado a prestar declaración testimonial si opta por guardar el secreto 
profesional, defiende la tesis de que el secreto profesional pertenece a la conciencia del abogado, por cuanto aquella actúa siempre por encima de los deseos de su cliente y no puede ser condicionada por ninguna otra regulación. Y, María Eugenia Talerico, abogada ex subdirectora de la UIF -Unidad de Información Financiera de Argentina-, aborda el delicado problema de El abogado ante las normas sobre lavado de dinero y financiación del terrorismo, advirtiendo que el esfuerzo conjunto del sector público y del sector privado puede contribuir de este flagelo que compromete el orden económico y la paz mundial. Respecto del delicado tema de los honorarios, la experimentada Rosalía Silvestre en $L a$ ética profesional y los honorarios del abogado, concluye que el camino no está claro:¿se puede legislar y aplicar lo que no ha sido pasible de un exhaustivo análisis y debate por parte de la abogacía?, y Marcelo Gobbi, en Honorarios mínimos y orden público: una perspectiva deontológica, examina desde una perspectiva ética las consecuencias de imponer mínimos obligatorios de los abogados, que considera inconsistente con los principios éticos. Suma también Abogacía interna en la Argentina, reflexiones a partir de una sentencia europea sobre confidencialidad de las comunicaciones entre clientes y abogados, comentando el caso Akzo (Tribunal Europeo de Justicia) y cuál podría ser su interpretación bajo las normas argentinas. Cierra Juan Manuel Vaquer, abogado con una extensa carrera corporativo que culmina como presidente de una de las más importantes empresas de la Argentina, aporta toda su experiencia en un breve, pero sólido Decálogo del abogado externo de empresas, estudio que transpira ética profesional en esta rama del ejercicio.

\section{¿Y los abogados jueces?}

Con tres trabajos muy interesantes, uno del magistrado Santiago Finn sobre La ética judicial como ética de las virtudes, y dos de Alejandro Turjansky termina la obra que comento.

En el primer estudio, Santiago Finn convoca al ideal profesional de los jueces que debe alejarse del perfil burocrático y normativista. En cuanto a los dos trabajos de Alejandro Turjanski, el primero un estudio comparativo de los códigos de ética judicial y una encuesta realizada entre magistrados, fueron preparados años atrás para enriquecer un libro sobre la ética judicial publicado por FORES, pero hemos entendido que dado el interés que mantienen debían complementar esta obra. El primero se refiere a su Encuesta a los jueces sobre temas de ética profesional, en tanto el segundo, titulado Estudio comparado de los códigos de ética judicial, aborda y compara distintos códigos de ética judicial sancionados en las provincias argentinas y otros de América Latina.

\section{Fores y los hombres y mujeres de derecho}

Considero que, con el libro que presento, la ONG convocante cumple una vez más con uno de los principios básicos de su fundación que considera que los hombres y mujeres del Derecho son el más importante factor de cualquier sistema judicial. La obra es un aporte objetivo con fundamentos fácticos y teóricos, suma al estudio de la ética y cómo está preparada para afrontar los retos presenta que la 'nueva' abogacía'. Y lo hace desde diversos enfoques institucionales y jurídicos con autores de muy distintas visiones y aún desde distintas localizaciones. Todo ello recomienda su lectura más de lo que el puedo incluir en este comentario. 